\title{
PERFIL E ANÁLISE DOS CASOS DE SUICÍDIO NOTIFICADOS NO MUNICÍPIO DE ARAGUAÍNA - TOCANTINS.
}

\author{
Profile and trend of suicide cases in the municipality of Araguaina Tocantins \\ Perfil y tendencia de casos de suicidio en el municipio de Araguaína Tocantins
}

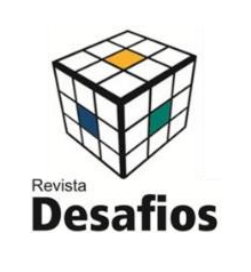

Artigo Original Original Article Artículo Original

Helierson Gomes ${ }^{1 *}$, Pamela Mayumi Kihara ${ }^{2}$, Stefani Morais Vieira ${ }^{2}$, Wathyson Alex de Mendonça Santos², Luiza Sousa Machado², Natã Silva dos Santos ${ }^{2}$, Andrielly Gomes de Jesus ${ }^{3}$.

${ }^{1}$ Doutorando Biologia Parasitária da Amazônia (UEPA). Laboratório de Saúde Coletiva, Curso de Graduação em Medicina, Universidade Federal do Tocantins, Araguaína - TO, Brasil.

${ }^{2}$ Graduando (a) em Medicina, Curso de Graduação em Medicina, Universidade Federal do Tocantins, Araguaína TO, Brasil.

3 Doutoranda Desenvolvimento Regional (UFT), Curso de Graduação em Medicina, Universidade Federal do Tocantins, Araguaína - TO, Brasil.

*Correspondência: Laboratório de Saúde Coletiva, Universidade Federal do Tocantins - Campus Centro de Ciências da Saúde (CCS), Curso de Graduação em Medicina, Universidade Federal do Tocantins, Araguaína TO, Brasil. Av. Dionísio Farias, 838 - Loteamento de Fátima| 77814-350 Araguaína/TO. e-mail profhelierson@gmail.com/helierson@mail.uft.edu.br.

\section{RESUMO}

Diante da atual epidemia de casos de suicídio entre a população mundial, esta pesquisa vem com objetivo de analisar o perfil e a tendência dos casos de suicídio na cidade de Araguaína Estado do Tocantins no período de 2008 a 2017. Estudo epidemiológico dos casos de suicídio foi realizado com base em métodos descritivo e ecológico realizado no município de Araguaína, localizado ao norte do Estado do Tocantins. No período estudado o município de Araguaína apresentou um total de 111 casos notificados de suicídio com uma significativa prevalência nos indivíduos do sexo masculino (79 \%), solteiros $(63,9 \%)$ com faixa etária entre 20 e 59 anos de idade com baixa escolaridade, sendo importante salientar que $61,2 \%$ dos casos de suicídio no município se tratam de pessoas que sequer terminaram o primeiro grau, sendo o enforcamento o método mais utilizado na execução do ato suicida $(60 \%)$, seguido pela intoxicação por pesticidas $(17 \%)$. Um ponto preocupante é a projeção de uma tendência crescente dos casos para os próximos anos, onde poderia agravar ainda mais a situação atual da cidade de Araguaína que apresenta uma taxa média anual moderada com pouco mais de 6 casos por 100 mil/habitantes.

Palavras-chave: Suicídio; Epidemiologia; Saúde mental.

\section{ABSTRACT}

In view of the current epidemic of suicide cases among the world population, this research aims to analyze the profile and trend of suicide cases in the city of Araguaína State of Tocantins from 2008 to 2017. An epidemiological study of suicide cases was carried out based on descriptive and ecological methods carried out in the municipality of Araguaína, located in the north of the State of Tocantins. During the period studied, the municipality of Araguaína had a total of 111 reported cases of suicide with a significant prevalence in males (79\%), single $(63.9 \%)$ aged between 20 and 59 years old with low education, it is important to note that $61.2 \%$ of suicide cases in the municipality are people who have not even finished primary school, with hanging being the most used method of executing the suicidal act $(60 \%)$, followed by pesticide poisoning $(17 \%)$. A worrying point is the projection of an increasing trend of cases for the coming years, where it could further aggravate the current 
situation in the city of Araguaína, which has a moderate annual average rate with just over 6 cases per 100 thousand / in habitants.

Keywords: Suicide; Epidemiology; Mental health.

\section{RESUMEN}

En vista de la epidemia actual de casos de suicidio en la población mundial, esta investigación tiene como objetivo analizar el perfil y la tendencia de los casos de suicidio en la ciudad de Araguaína, estado de Tocantins, de 2008 a 2017. Se realizó un estudio epidemiológico de casos de suicidio basado en Métodos descriptivos y ecológicos realizados en el municipio de Araguaína, ubicado en el norte del Estado de Tocantins. Durante el período estudiado, el municipio de Araguaína tuvo un total de 111 casos reportados de suicidio con una prevalencia significativa en hombres (79\%), solteros $(63.9 \%)$ con edades entre 20 y 59 años con baja educación, es importante tener en cuenta que el $61.2 \%$ de los casos de suicidio en el municipio son personas que ni siquiera han terminado la escuela primaria, siendo el ahorcamiento el método más utilizado para ejecutar el acto suicida (60\%), seguido del envenenamiento por pesticidas (17\%). Un punto preocupante es la proyección de una tendencia creciente de casos para los próximos años, donde podría agravar aún más la situación actual en la ciudad de Araguaína, que tiene una tasa promedio anual moderada con poco más de 6 casos por cada 100 mil / habitantes.

Descriptores: Suicidio; Epidemiología; Salud mental.

\section{INTRODUÇÃO}

O suicídio está entre as 20 causas de morte mais prevalentes no mundo, estando à frente de mortes por câncer de mama, malária, guerras e homicídio. Anualmente, cerca de 800 mil pessoas morrem por suicídio, sendo considerado a segunda causa de óbito entre jovens de 15 a 29 anos de ambos os sexos em todo o mundo, ficando atrás somente de acidentes de trânsito (WHO, 2018).

Historicamente, a concepção de suicídio passou por diversas mudanças. O termo origina-se da locução em latim "sui caedere" que significa "matarse”. Segundo André (2018), nota-se que o emprego do vocábulo é recente e uma de suas primeiras aparições foi ainda durante o século XVII, em uma das edições do Oxford English Dictionary de 1651, sendo intitulado muitas vezes antes como "morte voluntária" ou "morte intencional", conforme Ferreira (2008).

Quanto ao ato em si, sua constituição social, tradicionalmente, alterou-se inúmeras vezes ao longo dos séculos. Segundo Szasz (2002), na Antiguidade, não havia impreterivelmente deságio a respeito do suicídio, sendo, principalmente, a partir de Agostinho de Hipona, ou Santo Agostinho, no século V, que a prática passou a ter conotação pecaminosa, levandose em conta os preceitos judaico-cristãos vigentes na época. Adiante, ainda durante a Idade Média, em que Estado e Igreja mantinham fortes relações e interesses, o suicídio era considerado crime, levando à confiscação de bens e à punição do cadáver. No fim da Idade Média, com a separação entre Estado e Igreja, o poder médico e científico passou a deter certo controle sobre a sociedade, transferindo o ato de um conceito pecaminoso à patológico

No século XIX, Émile Durkheim analisa o suicídio não como um fenômeno psicológico individual, mas como um fato social, isto é, valores socioculturais que sobrepujam o indivíduo e podem exercer controle social. Assim, o sociólogo francês estabeleceu a Sociologia como uma ciência e como uma disciplina objetiva e delineou o suicídio como todo o "caso de morte que resulta, direta ou indiretamente, de um ato, positivo ou negativo, executado pela própria vítima, e que ela sabia que deveria produzir esse resultado" (RODRIGUES, 2019, p. 701). 
Hoje, além de um grave problema de saúde pública global, o suicídio constitui-se como importante objeto de estudo no campo da psicologia e psicanálise e no campo sociológico (WHO, 2018).

Levando-se em consideração as análises anteriores, as causas de um suicídio são complexas, podendo estar relacionadas a diversos fatores. Sejam eles acontecimentos recentes, como desemprego ou rompimento afetivo, ou motivações peculiares, tais como transtornos mentais, depressão, transtorno de humor bipolar, dependência química e esquizofrenia, os últimos estão presentes na maioria dos casos (BERTOLOTE E FLEISCHMANN, 2002).

Embora exista um vínculo bem instituídos entre distúrbios mentais e suicídio, segundo a Organização Mundial de Saúde (OMS), vários casos ocorrem em momentos de crise ou colapsos, como dores crônicas, crises de pânico ou problemas financeiros. Além disso pessoas em enfrentamento de conflitos, violência, desastres, abusos ou perdas, bem como próprias de grupos vulneráveis que sofrem discriminação, como imigrantes, refugiados, indígenas e pertencentes a comunidade LGBTI (Lésbicas, Gays, Bissexuais, Transgêneros e Intersexuais) e pessoas privadas de liberdade são fortemente associadas a comportamentos suicidas.

O suicídio, além de uma tragédia social e pessoal, é também um fator impactante na economia de forma que para cada suicídio seis outras pessoas são impactadas, tanto emocional, quanto economicamente (MACHADO E DOS SANTOS, 2015). Além disso, a economia também afeta diretamente a taxa de suicídio, lembrando que segundo Ferreira Junior (2015), 75\% dos suicídios se dão em países subdesenvolvidos ou em processo de desenvolvimento.

O suicídio é considerado um fenômeno que atinge todas as regiões do mundo. Globalmente, 79\% das mortes por suicídio ocorreram em países de baixa e média renda, onde $84 \%$ da população mundial vive. No entanto, os países de alta renda apresentaram a maior taxa - 11,5 para cada 100 mil habitantes (WHO, 2018).

O Brasil, por ser um país bastante populoso, possui um baixo índice de suicídio, no entanto está entre os dez países que registram os maiores números absolutos de suicídio no mundo. Em seis anos (20102016) a taxa de suicídio no Brasil aumentou 7\%, em contraposição ao índice global que teve queda de 9,8\%. Em 2010, foram contabilizados 5,7 suicídios a cada 100 mil habitantes, já em 2016, foram registrados 6,1 casos a cada 100 mil habitantes no país. Ainda nesse contexto, dentre os principais métodos utilizados para sua execução, encontram-se auto envenenamento com pesticidas, enforcamento e uso de armas de fogo (OMS, 2018).

No que se refere à distribuição geográfica, no Brasil, de 2002 a 2012, a região Norte se destaca com um aumento de $77,7 \%$ dos casos de suicídio, tendo os estados do Acre, Roraima, Tocantins e Amazonas duplicado suas ocorrências. Dados da região Nordeste também são alarmantes, com um aumento de 51,7\% dos casos no período. As regiões Centro-Oeste e Sul tiveram crescimento de $16,3 \%$ e $15,2 \%$ dos casos, respectivamente. Já na região Sudeste observou-se um acréscimo de $35,7 \%$ nas taxas de suicídio (FERREIRA JUNIOR, 2015).

$\mathrm{O}$ estado do Tocantins preocupa com o aumento dos casos nos últimos anos. De meados de 1998 a 2006, o Estado passou da 19a à $6^{a}$ posição nos índices de suicídio dos estados brasileiros, estando Palmas entre as primeiras colocadas dentre as capitais brasileiras (SENA-FERREIRA et al., 2014) e, segundo estudo realizado pelo Departamento de Informática do Sistema Único de Saúde - DATASUS (2016), o número de incidência em Palmas, representa mais de 50\% dos casos totais, sendo de 154 ocorrências na Capital para 352 gerais no 
Tocantins. Num comparativo entre 2016 e 2018, os casos de suicídio triplicaram no Estado (FILHO ESS et al., 2019).

Embora a Organização Mundial da Saúde (OMS) reconheça o suicídio como improrrogável obstáculo no quesito de saúde pública e disponha de políticas como "Mental Health Gap Action Programme (mhGAP)" (programa de saúde mental da OMS) que tem como objetivo a diminuição de $10 \%$ dos casos de suicídio nos países pertencentes aos Estados Membros da OMS, incluindo o Brasil, até 2020, ainda existem empecilhos como a efetividade de políticas públicas e a falta de informação, tornando-se substancial a promoção de estudos e análises dentro do tema pré-estabelecido de forma a colaborar com o enriquecimento científico e com a consumação de ações preventivas eficientes. (FILHO ESS et al, 2019). Respaldando-se nessa conjectura, este estudo possui como objetivo analisar o perfil e a tendência dos casos de suicídio na cidade de Araguaína Estado do Tocantins no período de 2008 a 2017.

\section{MATERIAIS E MÉTODOS}

O estudo foi realizado no Município de Araguaína, cidade localizada ao norte do estado do Tocantins, sua população estimada em 2018 era de 177517 habitantes, sendo assim o segundo mais populoso do estado, atrás apenas da capital Palmas. Apresenta uma densidade demográfica de 44 hab/ $\mathrm{Km}^{2}$ e IDH-M de 0,752 (IBGE, 2020).

O estudo epidemiológico dos casos de suicídio foi realizado com base em métodos descritivo e ecológico. O estudo contemplou $100 \%$ das notificações de casos confirmados de suicídio no município registrado na base de dados do Sistema de Informações de Vigilância Epidemiológica através da
Ficha de Notificação de suicídio no período de 2008 a 2017.

Os dados epidemiológicos estudados foram os índices de mortalidade por suicídio classificados por local/cidade de residência, ano de notificação, sexo, raça/cor, faixa etária e grau de escolaridade. $\mathrm{O}$ banco de dados utilizado para a caracterização da Análise da Distribuição Espaço-Temporal dos casos foi construído a partir das bases de dados do Sistema de informação de agravos notificados (SINAN) na Secretaria de Saúde Pública do estado do Tocantins.

Para análise foi utilizado o programa Microsoft ${ }^{\circledR}$ Excel® 2008 for Mac, versão 12.2.8, para a depuração dos dados epidemiológicos e análise descritiva da incidência dos casos de suicídio. Dentre as variáveis foi realizado a análise de variação (ANOVA) tendo como parâmetro de significância estatística quando o valor de $p$ for inferior a $5 \%$ e o modelo estatístico de Tendência exponencial para séries temporais $\left(Y_{t}=\beta_{0} * \beta_{1}{ }^{t} * e_{t}\right)$ para o período de cinco $\operatorname{anos}(2018$ a 2022) utilizando o software MINITAB 19.

\section{RESULTADOS E DISCUSSÃO}

No período estudado o município de Araguaína apresentou um total de 111 casos notificados de suicídio com uma significativa prevalência nos indivíduos do sexo masculino (79 $\%)$, solteiros $(63,9 \%)$ com faixa etária entre 20 e 59 anos de idade com baixa escolaridade, sendo importante salientar que $61,2 \%$ dos casos de suicídio no município se tratam de pessoas que sequer terminaram o primeiro grau (Tabela 1 ).

No que se refere a prevalência, o Brasil está entre os nove países com mais casos notificados. De 1998 a 2008, o índice de suicídios no país apresentou um aumento de $33,5 \%$, passando de 6.985 para 9.328 casos. Num contexto regional, a região Nordeste se 
destaca com dados alarmantes, tendo um aumento de $109 \%$ no número de mortes. Ademais, nas regiões Norte e Centro-Oeste o crescimento dos casos foi significativo, possuindo os estados do Tocantins, Acre e Amapá maior notoriedade na região Norte (VIDAL et al., 2014; WAISELFISZ, 2011).

Tabela 1. Análise dos casos de Suicídio em Araguaína Tocantins.

\begin{tabular}{|c|c|c|c|c|}
\hline Variáveis & & $\begin{array}{c}\mathbf{N} \\
(111)\end{array}$ & $\%$ & $p$ \\
\hline \multicolumn{5}{|l|}{ Sexo } \\
\hline Masculino & & 88 & 79 & \multirow{2}{*}{0.001} \\
\hline Feminino & & 23 & 21 & \\
\hline \multicolumn{5}{|c|}{ Faixa Etária } \\
\hline $10-19$ & & 5 & 4.5 & \multirow{5}{*}{0.0001} \\
\hline $20-39$ & & 47 & 42.4 & \\
\hline $40-59$ & & 40 & 36 & \\
\hline $60-79$ & & 15 & 13.5 & \\
\hline$>80$ & & 4 & 3.6 & \\
\hline Anos & de & & & \\
\hline \multicolumn{5}{|c|}{ Escolaridade } \\
\hline Nenhum & & 12 & 10.8 & \multirow{5}{*}{0.0001} \\
\hline 1 a 3 & & 31 & 27.9 & \\
\hline 4 a 7 & & 37 & 33.3 & \\
\hline 8 a 11 & & 22 & 19.8 & \\
\hline$>12$ & & 9 & 8.1 & \\
\hline \multicolumn{5}{|c|}{ Estado Civil } \\
\hline Solteiro & & 71 & 63.9 & \multirow{5}{*}{0.0001} \\
\hline Casado & & 26 & 23.5 & \\
\hline Viúvo & & 5 & 4.5 & \\
\hline Separado & & 6 & 5.4 & \\
\hline Outros & & 3 & 2.7 & \\
\hline
\end{tabular}

Fonte: GOMES H, et al., 2020.; SINAN, 2020.

Entre 1998 e 2008, o estado do Tocantins passou da $19^{\mathrm{a}}$ para a $6^{\mathrm{a}}$ posição nas taxas de maior incidência de suicídio, sendo preocupante o aumento de casos entre os indivíduos mais jovens (20 a 39 anos) do sexo masculino (FILHO et al., 2019).

Segundo Sadock (2017), as mulheres realizam três vezes mais tentativas de suicídio do que homens, apesar de existir um número maior de óbitos correspondente ao sexo masculino, onde esse fato pode estar associado à escolha do método, onde o enforcamento é o meio letal mais utilizado, corroborando com os achados do estudo em Araguaína.

Um ponto preocupante e muitas vezes negligenciado são os casos de suicídio nas lésbicas, gays, bissexuais, travestis, transexuais e intersexuais (LGBTI+). De uma forma histórica, a homossexualidade vai contra os padrões definidos pelo modelo social e, portanto, são condenados. Atualmente lutas contra a homofobia ganham destaque na sociedade, entretanto, o Brasil é um dos países que lidera assassinatos vinculados à LGBTfobia. Estima - se que a cada 19 horas, uma pessoa é vítima de assassinato ou suicídio (OLIVEIRA, 2018). De janeiro 2017 a maio do mesmo ano, foi confirmado um total de 153 mortos pela homotransfobia no Brasil, destes 42 foram por suicídio. (OLIVEIRA, 2018)

De acordo com Durkheim (2003), a sociedade exerce papel primordial na edificação do indivíduo. Logo, fatores sociais como a família e a escola, influenciam diretamente, tanto na produção, quanto na prevenção de um ato suicida. Sendo assim, estados civis que diminuem a integração social, como divorciado(a), viúvo(a) ou solteiro(a), são fatores que explicam a maior tendência suicida em ambos os sexos, em alguns estudos. Já outros apontam a confirmação dessa hipótese, majoritariamente, para o sexo masculino. (GONÇALVES et al., 2011; BRASIL, 2019).

De acordo com Calixto Filho, M., \& Zerbini, T. ,2016, a prevalência predominante nas tentativas de suicídio foi verificada entre as mulheres, que utilizam de métodos menos letais. Essa questão é coincidida com o fato de que elas são mais religiosas que os homens em geral, logo, podem ter, mais auxílio no enfrentamento de situações extremas como o suicídio. Além da religiosidade, a menor incidência do suicídio em mulheres tem sido atribuída à baixa prevalência do alcoolismo; às atitudes flexíveis em 
relação às aptidões sociais e ao desempenho de papeis durante a vida (GONÇALVES et al., 2011).

Contrapondo e agravando a situação, a dificuldade masculina em identificar o episódio suicida como intencional decorre de uma sociedade machista, devido não reconhecer o ato como oriundo de uma fragilidade emocional (Bahia et al., 2017). Além disso, a diferença entre os gêneros pode ser explicada a partir de um contexto histórico em que o homem tem mais acesso aos meios mais letais e pela menor exposição do sexo feminino adulto a sentimentos de falência frente ao insucesso financeiro. (Calixto Filho, M., \& Zerbini, T., 2016).

Além disso, em relação a ideação suicida, o baixo nível escolar é considerado um fator de risco, havendo estudos que estabelecem uma relação que quanto maior o grau de escolaridade menor o valor de ideação suicida e que a maior mortalidade por suicídio está concentrada nos grupos de menos escolaridade (VASCONCELOS-RAPOSO et al., 2016; SOUZA et al,. 2010; CORONEL et al., 2011;

LEE et al., 2009).

Lee et al. (2009) descobriram que a influência dos anos de permanência escolar no risco de suicídio pode ser explicada por alguns caminhos. Um deles é justificado pelo fato do indivíduo não ter sucesso profissional e não poder procurar um emprego melhor, impedindo-o de acumular riquezas materiais, tendo, assim, um maior risco de mortalidade por suicídio devido à menor segurança laboral e a associação com más condições monetárias, a exemplo da dívida. Outro caminho é que os mais bem sucedidos financeiramente possuem melhor acesso aos serviços de saúde, podendo levalos a prevenir ou controlar fatores de riscos proximais de morte por suicídio, como abuso de álcool e depressão.
Figura 1. Taxa média de óbitos por suicídio no Estado do Tocantins (2008 a 2017).

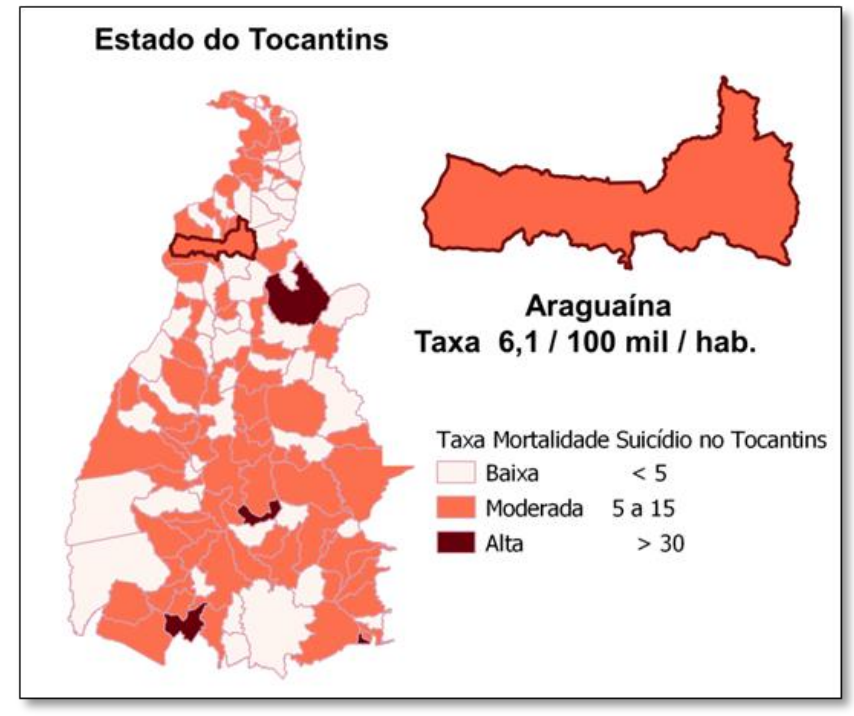

Fonte: GOMES H, et al, 2020.

De acordo com o ilustrado na Figura 1, Araguaína apresentou uma taxa de 6,1 casos por 100 mil habitantes, estando segundo a classificação da Organização Mundial de Saúde (OMS) no nível médio de taxa de mortalidade por suicídio entre 5 e 15/100 mil/hab (OMS, 2018).

Figura 2. Casos de suicídio segundo o método utilizado e sexo.

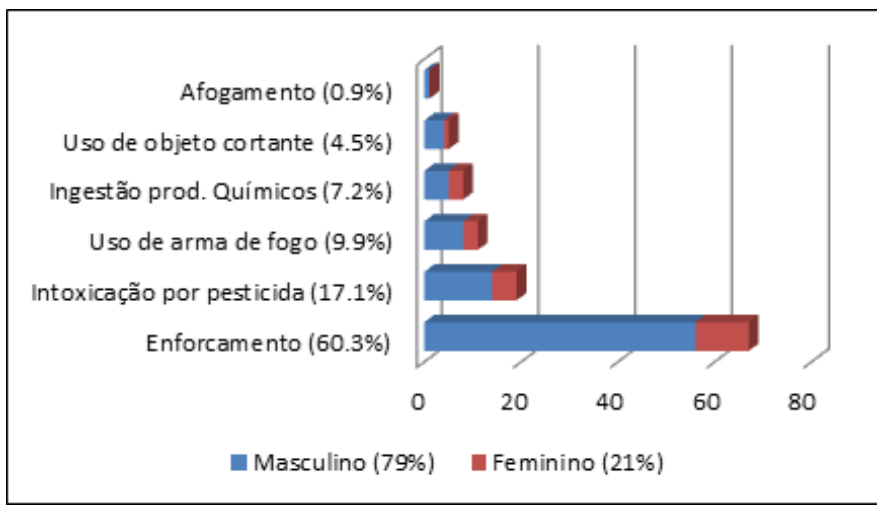

Fonte: GOMES H, et al, 2020.

O enforcamento foi significativamente $\mathrm{o}$ método mais utilizado na execução do ato suicida (60\%), seguido pela intoxicação por pesticidas (17\%), este apesar de não ser o mais incidente nos casos de mortalidade é segundo a OMS (2018), o 
mais prevalente nas notificações de tentativas de suicídio em todas as regiões do mundo (Figura 2).

A escolha do método demonstra que, em termos de intencionalidade, tanto os homens como as mulheres escolheram o enforcamento $(60.3 \%)$ como método que assegura a morte na tentativa, já que esse é o meio mais empregado no país. Óbitos com uso de pesticidas $(17.1 \%)$, tornaram-se o segundo método mais aplicado, uma problemática visto que, em sua grande maioria a sua comercialização é ilegal, ficando explicito que há uma fiscalização e um controle inadequado, assim como a quantidade de óbitos por arma de fogo (9.9\%) demostrando também que existe um fácil acesso a esse meio, mesmo sendo em grande parte, de comércios ilegais (MOREIRA, 2017; LOVISI, 2009; NOCK, 2008).

A figura 3 mostra que apesar de uma queda significativa dos casos no ano de 2014 os casos de suicídio apresentam uma projeção crescente dos casos nos últimos anos. D'eca Junior (2019) aponta que a Região Norte expressa o segundo maior índice de suicídio dentre as regiões brasileiras, em conformidade à crescente das taxas nacionais, crescimento esse acompanhado pelo município de Araguaína - TO, apresentando o segundo índice mais elevado dentre as cidades tocantinenses no ano de 2018 (FILHO et al., 2019).

Figura 3. Análise temporal dos Casos de Suicídio Araguaína - TO.

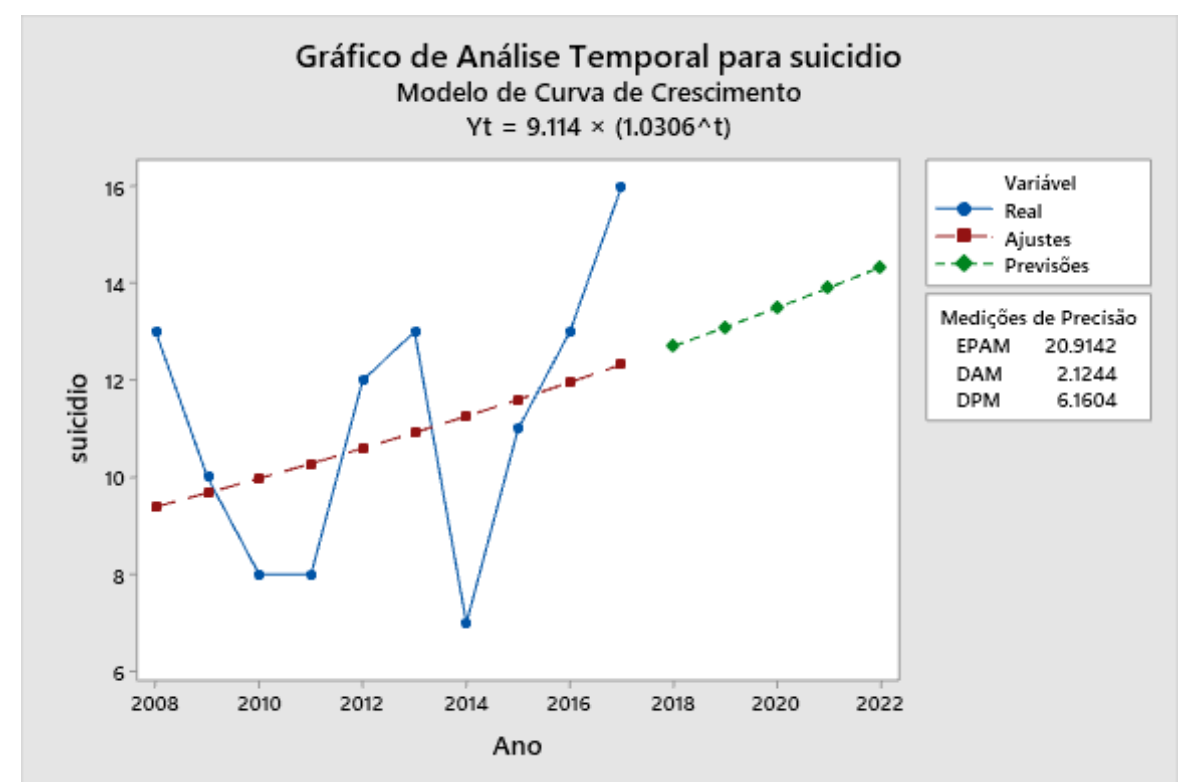

Fonte: GOMES H, et al, 2020.

Em contrapartida, constatou-se expressiva queda no ano de 2014, evidenciada por dados do Sistema de Informações sobre Mortalidades (SIM) no aumento dos óbitos relacionados a acidentes automobilísticos e àqueles de causa desconhecida em referência ao ano de 2013, enquanto a baixa na taxa de suicídio atingiu $63,6 \%$ (BRASIL, 2020) e possivelmente justificada pela subnotificação das mortes em decorrência de lesões autoprovocadas, sendo frequentemente atribuídas a outras razões (PINTO, 2017).

A partir do ano de 2015, as taxas de atentados suicidas retomaram a crescimento das taxas fundamentadas pela implementação da Portaria $\mathrm{N}^{\circ}$ 1.271/2014, que determina a notificação compulsória 
e imediata dos casos relacionados a lesões autoprovocadas, tendo prazo de $24 \mathrm{~h}$ após o conhecimento do ocorrido, com o intuito de prevenir a reincidência de atos de natureza autodestrutiva (BRASIL, 2020). Nessa perspectiva, atestou-se uma classificação mais adequada das mortes consoante a motivação, com declínio acentuado da menção de casos associados a causas acidentais ou de intenção indeterminada e incremento simultâneo dos suicídios.

Segundo dados da Organização Mundial da Saúde (OMS), não há uma causa ímpar para o suicídio, sendo a interação de fatores sociais, biológicos, psicológicos e culturais a razão mais usual para o comportamento suicida. Aspectos como isolamento, histórico de abuso de álcool e drogas, doenças físicas e perdas, sejam elas profissionais, financeiras ou de relacionamento, transtornos mentais, em especial a depressão, cada vez mais frequente na sociedade atual, quando associados, podem elevar os riscos de suicídio, ainda que não sejam a causa direta dele. Ademais, diversos casos ocorrem impulsivamente e, neste contexto, o fácil acesso aos meios, como instrumentos asfixiantes, armas de fogo e pesticidas, podem estar relacionados ao crescente número de eventos. (CDC, 2019; WHO, 2014).

\section{CONCLUSÃO}

Esta pesquisa apresenta resultados que corroboram com achados em vários outros estudos sobre o tema no Brasil e no mundo, onde uma maior prevalência de suicídio na população adulta jovem, solteiros, com predomínio do sexo masculino e baixa escolaridade.

Um ponto preocupante é a projeção de uma tendência crescente dos casos para os próximos anos, onde poderia agravar ainda mais a situação atual da cidade de Araguaína que apresenta uma taxa média anual moderada com pouco mais de 6 casos por 100 $\mathrm{mil} / \mathrm{hab}$.

Diante deste cenário e por se tratar de um problema de saúde pública, faz-se necessário promover estratégias e estudos a fim de analisar criticamente e fortalecer o funcionamento da rede assistencial de saúde mental para que os altos índices sejam revertidos.

Todos os autores declararam não haver qualquer potencial conflito de interesses referente a este artigo.

\section{REFERÊNCIAS}

ANDRÉ, W. Sobre o Conceito de Suicídio. In: Revista Estação Literária. (Volume 20/março). pp 154-174. Londrina. 2018.

BAHIA, C. A., AVANCI, J. Q., PINTO, L. W., \& MINAYO, M. C. DE S. (2017). Lesão autoprovocada em todos os ciclos da vida: Perfil das vítimas em serviços de urgência e emergência de capitais do Brasil. Ciencia e Saude Coletiva. https://doi.org/10.1590/1413-81232017229.12242017

BERTOLOTE, J.M., \& FLEISCHMANN, A. (2002). Suicide and psychiatric diagnosis. World Psychiatry.

BOTEGA, N.J. Comportamento suicida: epidemiologia. Departamento de Psicologia Médica e Psiquiatria da Faculdade de Ciências Médicas da Universidade Estadual de Campinas, Campinas, SP, Brasil. 2014 I volume 25 I número 3 I 231-236.

BRASIL. 2020. Suicídio - Histórias e taxas no Brasil e no mundo. Disponível em < https://www.psicologiamsn.com/2011/12/suicidiohistoria-brasil-mundo.html >

BRASIL. Boletim Epidemiológico. Ministério da Saúde. Perfil epidemiológico dos casos notificados de violência autoprovocada e óbitos por suicídio entre jovens de 15 a 29 anos no Brasil, 2011 a 2018. 2019. Disponível em: https://www.saude.gov.br/images/pdf/2019/setembro/ 13/BE-suic--dio-24-final.pdf.

BRASIL. Secretaria de Vigilância em Saúde. Ministério da Saúde (ed.). Saúde Brasil 2015/2016: Uma análise da situação de saúde e da epidemia pelo vírus Zika e por outras doenças transmitidas pelo Aedes aegypti. 2020. Disponível em: 
https://bvsms.saude.gov.br/bvs/publicacoes/saude br asil 20152016 analise zika.pdf.

BRASIL. Sistema de Informações Sobre Mortalidades. Ministério da Saúde. Óbitos por Causas Externas - Brasil. 2020. Disponível em: http://tabnet.datasus.gov.br/cgi/tabcgi.exe?sim/cnv/ex t10uf.def.

Centers for Disease Control and Prevention. Preventing Suicide. 2020. Disponível em: < https://www.cdc.gov/violenceprevention/suicide/fastf act.html >, Acesso em: 25 de maio. 2020.

Centers for Disease Control and Prevention. Risk Factors for Suicide. 2019. Disponível em: < https://www.cdc.gov/violenceprevention/suicide/risk protectivefactors.html >, Acesso em: 25 de maio. 2020.

CONSELHO FEDERAL DE PSICOLOGIA. O Suicídio e os Desafios para a Psicologia, Brasília: CFP, 2013. Disponível em: <https://site.cfp.org.br/wpcontent/uploads/2013/12/Su icidio-FINAL-revisao61.pdf>.

CORONEL, M. K., \& WERLANG, B.S.G. (2011). Resolução de problemas em homicidas e tentadores de suicídio. Boletim de Psicologia, 61(134), 103-116. Recuperado em 05 de abril de 2020, de http://pepsic.bvsalud.org/scielo.php?script=sci arttext \&pid=S0006-59432011000100009\&lng=pt\&tlng=pt.

D'EÇA J.A.; RODRIGUES L.S.; MENESES F.E.P.; COSTA L.D.L.N.; RÊGO A.S.; COSTA L.C.; BATISTA R.F.L. Mortalidade por suicídio na população brasileira, 1996-2015: qual é a tendência predominante?. Cad. saúde colet., Rio de Janeiro , v. 27, n. 1, p. 20-24, Mar. 2019. Disponível em: http://www.scielo.br/scielo.php?script=sci_arttext\&pi $\mathrm{d}=\mathrm{S} 1414462 \mathrm{X} 2019000100020 \& \operatorname{lng}=\mathrm{en} \& \mathrm{nrm}=\mathrm{iso}$

DURKHEIM, E. O suicídio. São Paulo: Martin Claret, 2003.

FERREIRA JUNIOR, A. O comportamento suicida no Brasil e no mundo. Revista Brasileira de Psicologia, 02(01), Salvador, Bahia, 2015. Disponível em: https://www.researchgate.net/publication/277130341 O_comportamento_suicida_no_Brasil_e_no_mundo The suicidal behavior in Brazil and in the worl $\underline{\mathrm{d}}$

FERREIRA, Renato Emanuel Campino. O Suicídio. Faculdade de Economia da Universidade de Coimbra, Coimbra, 2008. Disponível em: http://www4.fe.uc.pt/fontes/trabalhos/2008025.pdf.
FILHO, E.S.S.; CORREIA, L.C.S.; LIMA, P.R.; GOMES, H.; JESUS, A.G. O suicídio no Estado do Tocantins. Revista Eletrônica Acervo Saúde, v. 11, n. 12, p. e712, 8 jul. 2019. Disponível em: https://acervomais.com.br/index.php/saude/article/vie $\mathrm{w} / 712 / 513$.

GONÇALVES, L.R.C.; GONÇALVES, E.; OLIVEIRA JÚNIOR, L.B. Determinantes espaciais e socioeconômicos do suicídio no Brasil: uma abordagem regional. Nova Economia, v. 21, n. 2, p. 281-316, 2011. https://doi.org/10.1590/S0103$\underline{63512011000200005}$

LEE, W.; KHANG, Y.; NOH, M.; RYU, J.; SON, M.; HONG, Y. Trends in educational differentials in suicidemortality between 1993-2006 in Korea. Yonsei medical journal, v. 50, n. 4, p. 482-492, $2009 . \quad$ Disponível em: https://synapse.koreamed.org/search.php?where=avie w\&id $=10.3349 / \mathrm{ymj} .2009 .50 .4 .482 \&$ code $=0069$ YMJ $\underline{\text { vvmode }=\text { FULL }}$

LOVISI, G.M.; SANTOS, S.A.; LEGAY, L.; ABELHA, L.; VALENCIA, E. Análise epidemiológica do suicídio no Brasil entre 1980 e 2006. Rev. Bras. Psiquiatr., São Paulo, v. 31, supl. 2, p. S86-S93, Oct. 2009. Disponível em:http://www.scielo.br/scielo.php?script=sci_arttext \&pid=S15164446200900060007\&lng=en \&nrm=iso.

MACHADO, D.B. e SANTOS, D.N. Suicídio no Brasil, de 2000 a 2012. Jornal Brasileiro de Psiquiatria, v. 64, n. 1, p. 45-54, 2015. Disponível em:

https://www.scielo.br/scielo.php?pid=S00472085201 $\underline{5000100045 \& \text { script }=\text { sci_arttext } \& \text { tlng }=p t}$

MOREIRA, R.M.M.; FÉLIX, T.A.F.; FLÔR, S.M.C.; OLIVEIRA, E.N.; ALBUQUERQUE, J.H.M. Análise epidemiológica dos óbitos por suicídio. SANARERevista de Políticas Públicas, v. 16, 2017.Disponível em:https://sanare.emnuvens.com.br/sanare/article/vie $\underline{w F i l e / 1136 / 621}$

NOCK, M.K.; BORGES G.; BROMET E.J.; CHA C.B.; KESSLER R.C.; LEE S. Suicide and suicide behavior. Epidemiol Rev 2008; 30:133- 54. Disponível em: https://academic.oup.com/epirev/article/30/1/133/621 $\underline{357}$

OLIVEIRA, D.A.G. O suicídio na comunidade LGBT no brasil. 2018. 13 f. TCC (Graduação) Curso de Ciências Humanas, Universidade Federal de Juiz de Fora, Juiz de Fora, 2018. Disponível em: http://www.ufjf.br/bach/files/2016/10/DAIANAAPARECIDA-GOMES-DE-OLIVEIRA.pdf 
OMS. (Organização Mundial de Saúde). Folha informativa Suicídio. In OPAS Brasil. Disponível em:

https://www.paho.org/bra/index.php?option=com_co ntent $\&$ view=article \&id=5671:folha-informativasuicidio\&Itemid=839. 2018.

PINTO, L.L.T.; MEIRA, S.S.; RIBEIRO, Í.J.S.; NERY, A.A.; CASOTTI, C.A. Tendência de mortalidade por lesões autoprovocadas intencionalmente no Brasil no período de 2004 a 2014. J. bras. psiquiatr., Rio de Janeiro, v. 66, $\mathrm{n}$. 4, p. 203-210, Oct. $2017 . \quad$ Disponível em: http://www.scielo.br/scielo.php?script=sci_arttext\&pi $\mathrm{d}=\mathrm{S} 004720852017000400203 \& \operatorname{lng}=$ en $\& \mathrm{nrm}=$ iso

RODRIGUES, M.M.A. Suicídio e sociedade. Um estudo comparativo de Durkheim e Marx. Revista Latinoamericana de Psicopatologia Fundamental, São Paulo, v. 12, n. 4, p. 698-713, dez. 2009. Disponível em:

https://www.scielo.br/pdf/rlpf/v12n4/v12n4a06.

SADOCK, B.J.; SADOCK V.A.; RUIZ, P. Compêndio de psiquiatria: ciência do comportamento e psiquiatria clínica. 11 . ed. Porto Alegre: Artmed; 2017.

SENA-FERREIRA, N.; PESSOA, V.F.; BOECHATBARROS, R.; FIGUEIREDO, A.E.B.; MINAYO, M.C.S. Fatores de risco relacionados com suicídios em Palmas (TO), Brasil, 2006-2009, investigados por meio de autópsia psicossocial. Ciência e Saúde Coletiva. 2014. Disponível em: https://www.scielo.br/scielo.php?pid=S14138123201 $\underline{4000100115 \& \text { script }=\text { sci_abstract\&tlng }=\mathrm{pt}}$

SOUZA, L.D.D.M.; ORES, L.; DE OLIVEIRA, G.T.; CRUZEIRO, A.L.S.; SILVA, R.A.; PINHEIRO, R.T.,; HORTA, B.L. Ideação suicida na adolescência: Prevalência e fatores associados. Jornal Brasileiro de Psiquiatria. 2010. Disponível em:

https://www.scielo.br/scielo.php?script=sci arttext\&p $\mathrm{id}=\mathrm{S} 0047-20852010000400004$

VASCONCELOS-RAPOSO, J.; SOARES, A.R.; SILVA, F.; FERNANDES, M.G.; TEIXEIRA, C.M. Níveis de ideação suicida em jovens adultos. Estudos de Psicologia (Campinas). 2016. Disponível: https://www.redalyc.org/pdf/3953/395354131016.pdf

VIDAL, C.E.L.; GOMES, C.B.; MARIANO, C.A.; LEITE, L.M.R.; SILVA, R.A.; LASMAR, S.C. Perfil epidemiológico do suicídio na microrregião de Barbacena, Minas Gerais, no período de 1997 a 2012. Rio de Janeiro: Cad. Saúde Colet. 2014; 22 (2): 15864.
http://www.scielo.br/pdf/cadsc/v22n2/1414-462Xcadsc-22-02-00158.pdf

WAISELFISZ, J.J. Mapa da Violência 2011: Os jovens do Brasil. São Paulo, Brasília: Instituto Sangari, Ministério da Justiça (MS); 2011. Disponível em:

http://www.observatoriodeseguranca.org/files/Mapa \%20da\%20Viol\%C3\%AAncia\%202011\%20Os\%20j ovens $\% 20 \mathrm{do} \% 20 \mathrm{Brasil} . p d f$

WHO. Preventing suicide: A global imperative. 2014. Disponível em: < https://apps.who.int/iris/bitstream/handle/10665/1310 56/9789241564779 eng.pdf?sequence $=1$, Acesso em: 25 de maio. 2020.

WHO. Suicide in the world: Global Health Estimates. 2018.2 Disponível em: $<$ https://apps.who.int/iris/bitstream/handle/10665/32 6948/WHO-MSD-MER-19.3-eng.pdf > 